\title{
Flufenacet activity is affected by GST inhibitors in blackgrass (Alopecurus myosuroides) populations with reduced flufenacet sensitivity and higher expression levels of GSTs
}

\section{Research Article}

Cite this article: Dücker R, Parcharidou $E$, Beffa R (2020) Flufenacet activity is affected by GST inhibitors in blackgrass (Alopecurus myosuroides) populations with reduced flufenacet sensitivity and higher expression levels of GSTs. Weed Sci. 68: 451-459. doi: 10.1017/wsc.2020.54

Received: 24 December 2019

Revised: 11 May 2020

Accepted: 24 June 2020

First published online: 30 June 2020

\section{Associate Editor:}

Dean Riechers, University of Illinois

\section{Keywords:}

Ethacrynic acid; glutathione transferase; Group 15; herbicide resistance; RNA-seq; tridiphane; VLCFA

\section{Author for correspondence:}

Rebecka Dücker, Department of Crop Sciences, Plant Pathology and Crop Protection Division, Georg-August University Göttingen,

Grisebachstrasse 6, 37077 Göttingen, Germany. (Email: rduecke@gwdg.de)
(C) The Author(s), 2020. Published by Cambridge University Press on behalf of Weed Science Society of America. This is an Open Access article, distributed under the terms of the Creative Commons Attribution-

NonCommercial-ShareAlike licence (http:// creativecommons.org/licenses/by-nc-sa/4.0/), which permits non-commercial re-use, distribution, and reproduction in any medium, provided the same Creative Commons licence is included and the original work is properly cited. The written permission of Cambridge University Press must be obtained for commercial re-use.

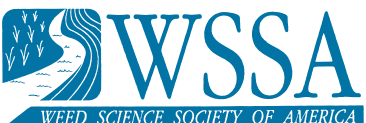

\section{Rebecka Dücker $^{1}$ (i), Evlampia Parcharidou ${ }^{2}$ and Roland Beffa ${ }^{3}$ (I)}

${ }^{1}$ Postdoctoral Researcher, Department of Crop Sciences, Plant Pathology and Crop Protection Division, GeorgAugust University Göttingen, Göttingen, Germany; ${ }^{2}$ Graduate Student, Department of Crop Sciences, Plant Pathology and Crop Protection Division, Georg-August University Göttingen, Göttingen, Germany and ${ }^{3}$ Team Leader, Bayer AG, Crop Science Division, Frankfurt/Main, Germany

\begin{abstract}
The WSSA Group 15 (HRAC Group $\mathrm{K}_{3}$ ) herbicide flufenacet is a key compound in weed resistance management, primarily used for PRE control of grass weeds in winter cereal-based crop rotations in Europe. Although resistance to compounds of its mechanism of action (inhibition of the synthesis of very-long-chain fatty acids) generally evolves slowly, reduced flufenacet efficacy due to enhanced glutathione transferase (GST) activity has been described in several blackgrass (Alopecurus myosuroides Huds.) populations. The present study aimed to better understand of the mechanism of flufenacet detoxification in A. myosuroides. Therefore, we characterized four A. myosuroides populations with different levels of flufenacet sensitivity. Flufenacet degradation was significantly slowed down in a sensitive population and a population with reduced flufenacet sensitivity by the use of the GST inhibitors tridiphane and ethacrynic acid at sublethal rates. Finally, an RNA sequencing (RNA-seq) study with the four A. myosuroides populations was conducted. In total, six differentially expressed GSTs and nine transcription factors as well as a keto-acyl-CoA reductase involved in the biosynthesis of very-long-chain fatty acids were identified as candidate genes among a set of 319 significantly more highly expressed gene-associated contigs. Among a set of 218 contigs with significantly lower expression levels, receptor kinase activity was the most frequent annotation. In summary, the likely GST-mediated reduction in sensitivity evolves in A. myosuroides at a slow rate and can partially be reversed by an interaction between flufenacet and the GST inhibitors tridiphane and ethacrynic acid. This provides further evidence for enhanced GST activity as a key mechanism in flufenacet resistance in A. myosuroides and supports the hypothesis that the six differentially expressed GSTs detected in the present RNA-seq study are potentially involved in flufenacet resistance.
\end{abstract}

\section{Introduction}

Weeds play a major role in lowering productivity in arable fields, and their control has been a major task in farming since the beginning of agriculture. The availability of cost-effective and resource-saving organic herbicides has led to a shift from cultural and mechanical control to intensive utilization of chemical weed control since the middle of the last century. However, besides increasingly restrictive pesticide registration processes, emerging cases of herbicideresistant weeds restrict the variety of herbicides available in different cropping systems and limit the number of possible rotations of active ingredients in the sense of good agricultural practice and optimal weed management.

Blackgrass (Alopecurus myosuroides Huds.) is one of the most noxious weeds in temperate Europe. Particularly in winter cereal-dominated cropping areas, A. myosuroides infestations can be severe, and due to this weed's evolution of herbicide resistance to several chemical classes, effective control has become increasingly complex. To date, resistance to seven herbicide mechanisms of action (MoAs) has been described in A. myosuroides, occasionally occurring in multiple-resistant populations (Heap 2019). Of particular concern, resistance to herbicides predominantly used in POST applications, such as inhibitors of acetyl-CoA synthase (WSSA Group 1, HRAC Group A) and acetolactate synthase (WSSA Group 2, HRAC Group B), is becoming more widespread (Peterson et al. 2018). Therefore, ensuring satisfactory efficacy of PRE herbicides by keeping the number of $A$. myosuroides plants at least below the estimated economic thresholds of 16 to 33 plants $\mathrm{m}^{-2}$ (Mennan et al. 2003) has become increasingly important (Bailly et al. 2012). However, higher control rates may be desirable to keep seedbanks low, particularly in cases of known resistance. 
As farmers increasingly rely on flufenacet, an inhibitor of the synthesis of very-long-chain fatty acids (VLCFAs), for the control of A. myosuroides (Hull and Moss 2012), the selection pressure on the treated populations has also increased. Although resistance to this herbicide MoA is considered to evolve slowly (Moss et al. 2019), some A. myosuroides populations have shown the potential to evolve resistance against inhibitors of VLCFA synthesis (Dücker et al. 2019b; Hull and Moss 2012; Rosenhauer and Petersen 2015). In A. myosuroides populations from northern Germany and several Lolium spp. populations, flufenacet was shown to be detoxified by glutathione conjugation (Dücker et al. 2019a, 2019b). Flufenacet tolerance in crops such as maize (Zea mays L.) was also linked to enhanced glutathione transferase (GST) activity (Bieseler et al. 1997). Yet neither the regulation of resistance genes nor individual isoforms detoxifying flufenacet have been described. GSTs, however, have been identified as enzymes that detoxify various other herbicides such as alachlor, atrazine, EPTC, or metolachlor (Brabham et al. 2019; Evans et al. 2017; Gronwald and Plaisance 1998; Timmerman 1989). This type of metabolism-based herbicide resistance can lead to unpredictable cross-resistance to other herbicide MoAs and chemical classes (Yu and Powles 2014) and can even confer resistance to herbicides not yet on the market (Busi et al. 2012). As a result, fewer herbicides have become available for effective weed control, making the choice of a suitable compound more challenging. In addition, potential reductions of the registered field rates in Europe may increase the risk of evolution of non-target site resistance (Neve and Powles 2005) and survival of populations already showing a shift in sensitivity to flufenacet in the field.

The use of compounds described as GST inhibitors can partially reverse resistance to herbicides detoxified by GST activity. Several compounds, including ethacrynic acid and tridiphane, were identified as inhibitors of plant GSTs (Ezra et al. 1985; Lamoureux and Rusness 1986). Various GST inhibition studies were conducted with different compounds. These were found to be active on isoforms identified in crops such as maize or soybean [Glycine max (L.) Merr.] (Li et al. 2017; Skipsey et al. 1997), but also on isoforms identified in weeds, such as AmGSTF1, a phi class GST frequently associated with metabolism-based resistance in A. myosuroides. It has been suggested to play a key role in signaling response to abiotic stress (Cummins et al. 2013; Tétard-Jones et al. 2018). However, GST inhibitors have not yet been tested in combination with flufenacet.

In this study, we characterized four A. myosuroides populations with different levels of flufenacet sensitivity in a dose-response bioassay. We compared ${ }^{14} \mathrm{C}$-flufenacet degradation rates in two populations that exhibit contrasting responses to the herbicide in the absence and presence of different GST-inhibiting compounds and finally identified differentially expressed GSTs as candidate genes in an RNA sequencing (RNA-seq) experiment.

\section{Materials and Methods}

\section{Dose-Response of Different Alopecurus myosuroides Populations to Flufenacet}

Four populations were selected for a dose-response bioassay: the sensitive A. myosuroides populations 'Herbiseed-S' (Herbiseed, Twyford, UK) and 'Appel-S' (Appels Wilde Samen, Darmstadt, Germany); an A. myosuroides field population from the northern Germany marsh region of Kehdingen (Kehdingen1), which was previously described to be significantly less susceptible to flufenacet due to enhanced metabolism (Dücker et al. 2019b); and another population from the same region, suspected to be more difficult to control (Kehdingen2). The seedlings were sown in tissue culture containers (MP Biomedicals, Eschwege, Germany) on $0.7 \%$ agar Type A (Sigma-Aldrich, Steinheim, Germany) containing $\mathrm{KNO}_{3}$ (Sigma-Aldrich) at a concentration of $0.02 \mathrm{M}$. The seeds were kept in the dark at $4 \mathrm{C}$ for $5 \mathrm{~d}$ and were then placed in a greenhouse for germination. The greenhouse was set to $22 / 16 \mathrm{C}$ day/night conditions with a 14 -h photoperiod provided by Master HPI-T plus 400W/645 E40 metal-halide lamps (Philips, Amsterdam, The Netherlands) at approximately 200 $\mu \mathrm{mol} \mathrm{m}{ }^{-2} \mathrm{~s}^{-1}$. As the primordial root emerged, 15 seedlings per population and treatment were transplanted into pots containing sandy loam with $2.2 \%$ organic matter (three pots with five seedlings each). The seedlings were covered with a thin layer of coarse sand and treated on the same day with $500,250,125,62.5,31.3,15.6,7.8$, 4.0, and $0 \mathrm{~g}$ flufenacet $\mathrm{ha}^{-1}$ formulated as $\mathrm{Cadou}^{\circledR} \mathrm{SC}$ using a laboratory track sprayer with a single flat spray nozzle (Tee)et ${ }^{\bullet}$ nozzle XR8001, $300 \mathrm{~L} \mathrm{ha}^{-1}, 200 \mathrm{kPa}$ ). The sprayed seedlings were irrigated from above after treatment and grown for $3 \mathrm{wk}$ under the previously described greenhouse conditions. The aboveground fresh weight was assessed, and the dose-response data were analyzed as a completely randomized experiment using the three-parameter log-logistic model of the DRC package in R software (RStudio v. 3.5.0) (Ritz et al. 2015). The equation with parameters $b$ (relative slope around the inflection point), $d$ (upper limit), and $e$ (inflection point) was used (Equation 1). The experiment was repeated once.

$$
f(x)=\frac{d}{1+\exp \{b[\log (x)-\log (e)]\}}
$$

\section{Flufenacet Degradation in Alopecurus myosuroides Seedlings with High and Reduced Flufenacet Sensitivity after Treatment with Selected GST Inhibitors}

The seedlings of the populations Herbiseed-S, Appel-S, Kehdingen1, and Kehdingen2 were grown in tissue culture containers as described earlier until the first leaf reached about 2.5 $\mathrm{cm}$ in length. Initially, a phytotoxicity test was conducted to determine symptom-free dose rates of flufenacet and the GST inhibitors tridiphane (Dr. Ehrenstorfer, Augsburg, Germany), chloro-7nitrobenz-2-oxa-1,3-diazole (NBD-Cl, Sigma-Aldrich), triphenyltin chloride (TPT-Cl, abcr GmbH, Kalsruhe, Germany), ethacrynic acid (Alfa Aesar, Wand Hill, MA, USA), and diethyl maleate (Acros Organics, Beijing, China), as well as malathion, a frequently used inhibitor of cytochrome P450 monooxygenases (CYPs; Sigma-Aldrich). Then, 32 seedlings per inhibitor treatment and population were incubated in $20-\mathrm{ml}$ scintillation vials containing $1.2 \mathrm{ml}$ of commercial water $\left(\mathrm{Volvic}^{\mathrm{m}}\right)$ with $\mathrm{KNO}_{3}$ at a concentration of $0.02 \mathrm{M}$ and ${ }^{14} \mathrm{C}$-radiolabeled flufenacet at a concentration of $15 \mu \mathrm{M}$ and $16.7 \mathrm{kBq} \mathrm{ml}^{-1}$. Treatments were tridiphane $(10 \mathrm{mM})$, NBD-Cl $(10 \mu \mathrm{M})$, triphenyltin chloride $(1 \mu \mathrm{M})$, ethacrynic acid $(1 \mathrm{mM})$, diethyl maleate $(100 \mu \mathrm{M})$, and malathion $(10 \mathrm{mM})$, with a flufenacet-only control. These 32 seedlings were incubated for 24 $\mathrm{h}$ in a growth chamber at $22 / 16 \mathrm{C}$ day/night conditions with a $14-\mathrm{h}$ photoperiod provided by Master TL-D 58W/840 REFLEX fluorescent lamps (Philips) at approximately $400 \mu \mathrm{mol} \mathrm{m}{ }^{-2} \mathrm{~s}^{-1}$. The seedlings were washed two times in water and one time in $50 \%$ acetone. Four times for each treatment, including the control, eight of these 32 seedlings were pooled to one sample to obtain sufficiently high signals, leading to four pooled samples in total. These were 
extracted and analyzed by high-performance liquid chromatography as described by Dücker et al. (2019a). The percentages of metabolized flufenacet recovered from differently treated seedlings were analyzed with a Kruskal-Wallis one-way ANOVA on ranks using SigmaPlot v. 13.0 (Systat Software, San José, CA, USA). Differences between two populations with the same treatment were analyzed using a Mann-Whitney test included in the R software (RStudio v. 3.5.0). The experiment was repeated once, and the data were pooled for the analysis.

\section{Illumina Transcriptome Sequencing of Alopecurus myosuroides Seedlings with High and Reduced Flufenacet Sensitivity}

For an RNA-seq study, the seeds of the populations Herbiseed-S, Appel-S, Kehdingen1, and Kehdingen2 were sterilized for $5 \mathrm{~min}$ in $5 \%$ sodium hypochlorite and rinsed five times with sterile demineralized water. The seeds were dried on filter paper and sown in tissue culture containers (MP Biomedicals) under sterile conditions. The containers were filled with $30 \mathrm{ml}$ of sterile $2-\mathrm{mm}$ glass beads, $30 \mathrm{ml}$ of sterile 4 - $\mathrm{mm}$ glass beads, and $12 \mathrm{ml}$ of sterile commercial water $\left(\right.$ Volvic $^{\mathrm{TM}}$ ) with $\mathrm{KNO}_{3}$ at a concentration of $0.02 \mathrm{M}$. The containers were kept in the dark at $4 \mathrm{C}$ for $5 \mathrm{~d}$ and were then transferred into a climate chamber under the conditions described earlier. The seedlings were grown until the first leaf reached a length of about $2.5 \mathrm{~cm}$. To assess constitutive differences between the biotypes, the untreated seeds were removed from the containers, and each six individual whole seedlings per population were immediately frozen at $-80 \mathrm{C}$.

The frozen seedlings were ground in a Tissue Lyser II swing mill (Qiagen, Hilden, Germany) at $30 \mathrm{~Hz}$ for $30 \mathrm{~s}$ in 2-ml reaction tubes each containing three tungsten carbide beads $(3 \mathrm{~mm})$. The ground tissue was suspended in standard buffer (RLT) provided with the Qiagen RNeasy Kit (Qiagen), and total RNA extraction was performed according to the manufacturer's instructions, including an on-column DNase treatment with an RNase-Free DNase Set (Qiagen). High-quality RNA was assured (RIN scores $>7$ ) using the RNA 6000 Nano Kit (Agilent Technologies, Waldbronn, Germany) as defined in the manufacturer's instructions. cDNA libraries were obtained using the Illumina TruSeq Stranded mRNA Library Prep kit (Illumina, San Diego, CA, USA). The multiplexed cDNA libraries were sequenced using an Illumina HiSeq 4000 sequencer (Illumina). Each library was measured on three lanes to obtain paired-end reads of 150 -bp length in high-output mode, providing three technical replicates per biological replicate.

\section{RNA-seq Analysis}

The reads were demultiplexed using an in-house script by Fasteris (Geneva, Switzerland). The obtained paired-end reads were trimmed and mapped against an A. myosuroides reference transcriptome assembled with Illumina reads by Gardin et al. (2015) using BWA with the maximal exact matches (MEM) algorithm (Li 2013) (BWA v. 7.12) within the Genedata Expressionist Refiner Genome software (v. 9.5; Genedata, Basel, Switzerland). The reads were trimmed mean of $\mathrm{M}$ values (TMM) normalized in order to robustly equate the overall expression levels of genes between samples under the assumption that the majority of them are not differentially expressed (Robinson and Oshlack 2010). A pairwise differential gene expression analysis was conducted using the exact test of edgeR provided by the Blast2GO PRO software v. 4.1 .9 (Conesa et al. 2005). Candidate genes were selected with the following cutoff criteria: false discovery rate

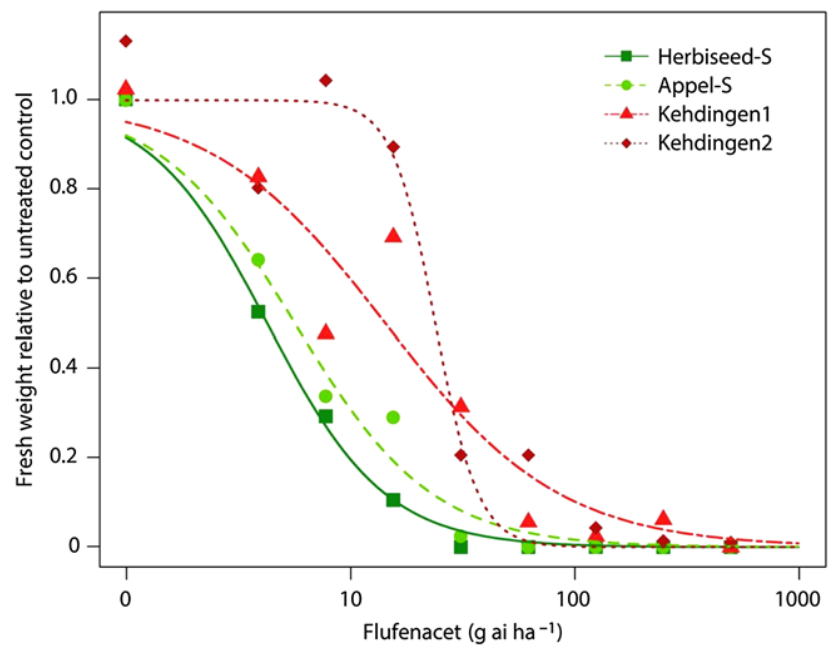

Figure 1. Dose-response analysis of the fresh weight of four Alopecurus myosuroides populations treated with different dose rates of flufenacet estimated using a threeparameter log-logistic model (see Equation 1).

(FDR) $\leq 0.05$ and log fold-change $\geq 2$. The probable function of the candidate genes was annotated using the Basic Local Alignment Search Tool X (BLASTx; Camacho et al. 2009) against the National Center for Biotechnology Information non-redundant database. Protein sequence similarities between the candidate contigs and AmGSTF1 (Cummins et al. 2013) were determined through global pairwise alignments using the EMBOSS Needle algorithms (https://www.ebi.ac.uk/Tools/psa/emboss_needle).

\section{Results and Discussion}

To better understand the mechanisms of flufenacet detoxification in A. myosuroides, we used dose-response bioassays to characterize the four A. myosuroides populations, Herbiseed-S, Appel-S, Kehdingen1, and Kehdingen2 (Figure 1). The sensitive reference populations Herbiseed-S and Appel-S had effective dose rates 50 $\left(\mathrm{ED}_{50}\right.$ values $)$ of $4.3 \pm 0.8(\mathrm{P}<0.01)$ and $5.7 \mathrm{~g} \pm 1.3(\mathrm{P}<0.01)$ flufenacet $\mathrm{ha}^{-1}$, respectively. They were more susceptible than the northern Germany field populations Kehdingen1 and Kehdingen2, which had $\mathrm{ED}_{50}$ values of $14.4 \pm 3.4(\mathrm{P}<0.01)$ and $24.0 \mathrm{~g} \pm 2.2(\mathrm{P}<0.01)$ flufenacet $\mathrm{ha}^{-1}$, respectively. A 5.6 -fold increase in herbicide rate required to inhibit growth by $50 \%$ was calculated for population Kehdingen2. The sensitivity of this population to flufenacet was significantly lower than the sensitivity of the reference populations Herbiseed-S and Appel-S. Kehdingen1, however, only differed significantly from Herbiseed-S. Sufficient control with the field rate of $250 \mathrm{~g}$ flufenacet ha ${ }^{-1}$ was indicated with $\mathrm{ED}_{90}$ values between 16.2 and $102.9 \mathrm{~g}$ flufenacet $\mathrm{ha}^{-1}$ for all populations tested under favorable conditions. This relatively low level of reduction in sensitivity is in accordance with previously published studies (Dücker et al. 2019b; Hull and Moss 2012; Rosenhauer and Petersen 2015) and may result from crossresistance to other herbicide chemistries or may directly be selected by flufenacet applications. Reduced sensitivity to various herbicides was previously described for populations from the marsh regions of northern Germany, the origin of the populations characterized in this study (Dücker et al. 2019b; Rosenhauer and Petersen 2015) and may result from non-target site resistance.

Enhanced metabolism has previously been found as the dominant form of non-target site resistance to flufenacet in 
(a)

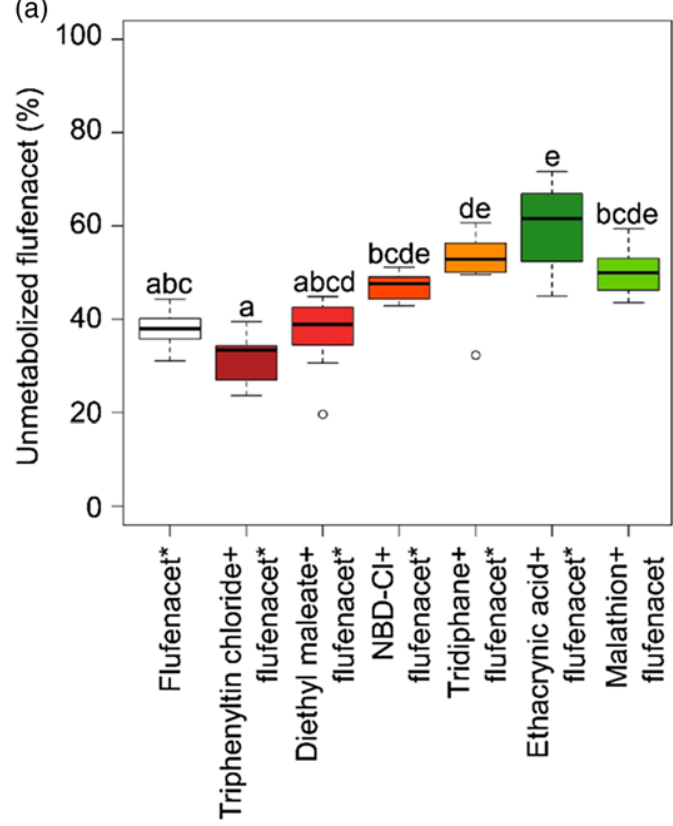

(b)

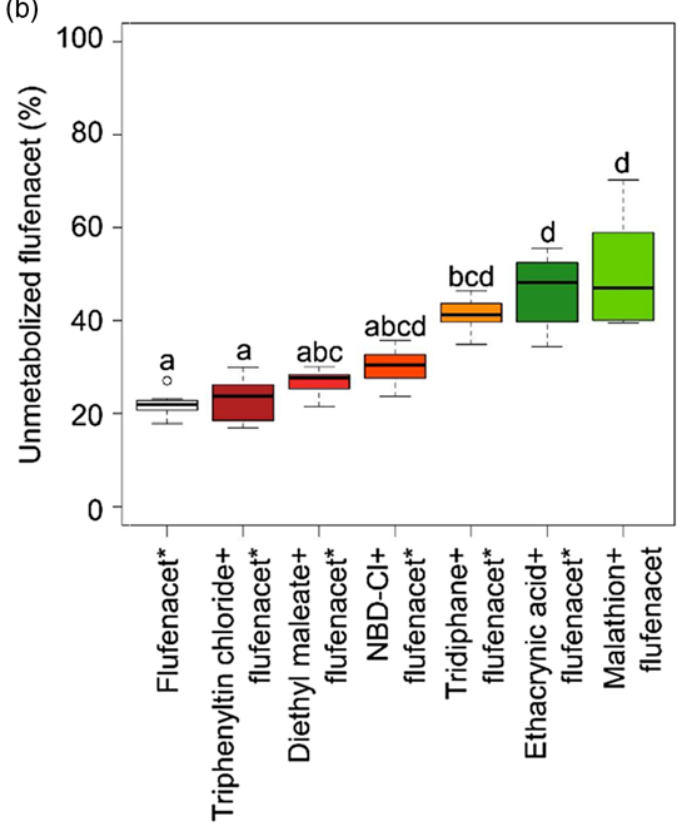

Figure 2. Flufenacet degradation $24 \mathrm{~h}$ after treatment with different inhibitors in the sensitive Alopecurus myosuroides population Herbiseed-S (A) and population Kehdingen1 with reduced flufenacet efficacy (B). Different letters indicate significant differences in flufenacet degradation between treatments, and asterisks $\left({ }^{\star}\right)$ indicate significant differences between the two populations for each treatment $(P \leq 0.05)$.

A. myosuroides as well as in Lolium spp. (Dücker et al. 2019a, $2019 b$ ). The results of the experiment with different potentially synergistic inhibitors in this study point in the same direction. To test the effects of different chemicals on the performance of flufenacet, seedlings of the sensitive A. myosuroides population Herbiseed-S and the less susceptible field population Kehdingen 1 were treated with ${ }^{14} \mathrm{C}$-radiolabeled flufenacet in the absence and presence of different inhibitors of GSTs and the insecticide and inhibitor of CYPs and acetylcholinesterase malathion. After 24 $\mathrm{h}$, on average, $37.9 \%$ of unmetabolized flufenacet was recovered from the sensitive population Herbiseed-S and $21.9 \%$ of unmetabolized flufenacet was recovered from seedlings of the less susceptible population Kehdingen1, treated with flufenacet only (Figure 2). Based on a $t$-test $(\mathrm{P} \leq 0.05)$, population Kehdingen1 degraded flufenacet significantly faster than population Herbiseed-S in all treatments, except for the treatment with malathion.

A comparison of flufenacet degradation rates revealed that the detoxification in the populations Herbiseed-S and Kehdingen 1 was affected to different extents by treatments with selected GST inhibitors and the CYP inhibitor malathion (Figure 2). Only seedlings of population Herbiseed-S treated with triphenyltin-chloride degraded flufenacet on average faster than seedlings of this population treated with flufenacet only. In all other treatments, the average degradation rate was slower in comparison to treatments with flufenacet only. While the differences were not statistically significant for seedlings treated with diethyl maleate and NBD-Cl, slower flufenacet degradation resulting from treatments with the GST inhibitors tridiphane and ethacrynic acid was statistically supported by a Kruskal-Wallis test. Interestingly, treatments with the CYP inhibitor malathion also led to significantly reduced flufenacet degradation rates in population Kehdingen1, although hydroxylated phase I metabolites were not found in flufenacettreated A. myosuroides (Dücker et al. 2019b). Similar inhibitory effects were also described for Lolium spp. (Parcharidou 2019) and in a recombinant phi-class GST originating from maize. In addition, the authors hypothesized that malathion binds to the substrate binding site of the tested GST isoform (Kapoli et al. 2008). Diverse GST-inhibitory effects of malathion have previously been observed in other organisms such as rats (Hazarika et al. 2003). However, further investigations are required to clarify whether the observed effect is a result of direct inhibition of GSTs or due to other effects of malathion.

In general, these results demonstrate the presence of an interaction between GST inhibitors and flufenacet as previously described for other herbicides detoxified by GSTs (Cummins et al. 2013; Ezra et al. 1985). Moreover, they support previous findings by Dücker et al. (2019a, 2019b) suggesting enhanced GST activity as a key driver for a reduction in flufenacet sensitivity. The use of GST inhibitors as synergists for field applications, however, is generally problematic, as compounds such as ethacrynic acid inhibit GST classes expressed in humans, for example, alpha or pi class (Huang et al. 2015) as well as plant-specific GSTs such as isoforms belonging to the phi or tau class.

While the inhibition of flufenacet degradation by tridiphane and ethacrynic acid consolidates the formulated hypothesis that flufenacet is detoxified by isoform(s) belonging to the superfamily of GSTs, it is not clear which isoforms of which class(es) are involved in the reduction of flufenacet sensitivity. Altogether 47 GST isoforms belonging to the classes tau (28), phi (13), theta (3), zeta (2), and lambda (2) were identified in the genome of Arabidopsis thaliana (Wagner et al. 2002), and 79 putative GSTs belonging to the classes tau (52), phi (17), zeta (4), DHAR (2), EF1G (2), theta (1) and TCHQD (1) were identified in rice (Oryza sativa L.) (Jain et al. 2010). The large number of GST isoforms, as well as sequence similarities, complicates the identification of candidate GSTs in A. myosuroides with traditional methods like real-time PCR. This may particularly apply to the larger classes tau and phi, which have probably undergone extensive gene-duplication events after divergence of monocotyledonous and dicotyledonous plants (Monticolo et al. 2017). Therefore, an 
(a)

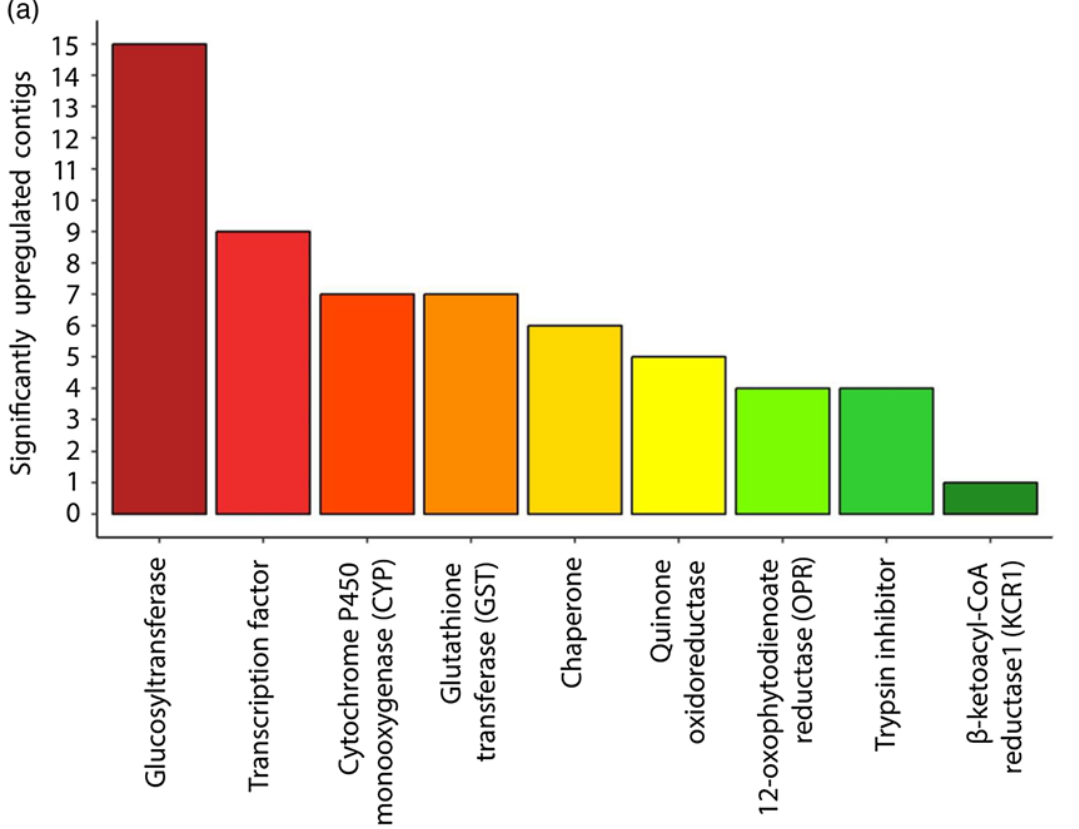

(b)

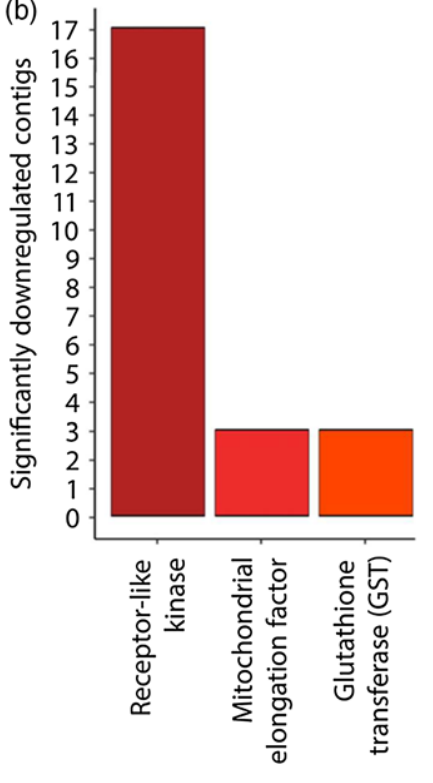

Figure 3. Most frequent BLASTx annotations of contigs with differential expression. (A) Most frequent annotations with higher expression levels in Alopecurus myosuroides populations from Kehdingen, Germany, including a $\beta$-ketoacyl-CoA reductase1 (KCR1); and (B) most frequent annotations with higher expression levels in $A$. myosuroides populations from Kehdingen.

RNA-seq approach was chosen for identification of candidate genes conferring reduction in flufenacet sensitivity in A. myosuroides by investigating differences in constitutive gene expression among populations with a shift in sensitivity.

A differential gene expression analysis was used to identify transcriptomic differences between the sensitive populations Herbiseed-S and Appel-S and the less susceptible populations Kehdingen1 and Kehdingen2. This revealed 319 gene-associated contigs with significantly higher expression and 218 gene-associated contigs with significantly lower expression as potential candidate genes conferring differences in flufenacet susceptibility. Annotations of detoxification-associated functions were most abundant among the set of more highly expressed contigs, indicating a constitutive upregulation of detoxification pathways. In addition to 15 glucosyltransferases and 7 CYPs, 6 GSTs (7 contigs) were found among the most frequent annotations (Figure 3). Furthermore, 9 significantly more highly expressed contigs were annotated as different transcription factors. Additionally, further transcription- and translation-related functions such as zinc finger domains, several kinases, a ribosome-binding factor, a mitochondrial RNA helicase, and chaperones were significantly more highly expressed. The higher expression of this group of genes indicates a complex change in gene expression patterns in the less sensitive populations that may play a role in the reduced sensitivity in the populations of interest.

Interestingly, one of the significantly more highly expressed contigs, although with relatively low TMM values, was annotated as $\beta$-ketoacyl-CoA reductase1 (KCR1). KCR1 is part of the VLCFA elongase complex, which additionally consist of a enoyl-CoAreductase, a $\beta$-hydroxyacyl-CoA dehydratase, and ketoacyl-CoAsynthases, the family of enzymes that represents the putative site of action of flufenacet and catalyze the first and rate-limiting step of the elongation of VLCFAs (Haslam and Kunst 2013; Trenkamp et al. 2004). This observation leads to the hypothesis that a higher production rate of VLCFAs may lead to reduced sensitivity of weed populations treated with herbicides inhibiting the synthesis of VLCFAs. The combination of higher flufenacet detoxification rates and possibly a change in the VLCFA elongation complex can lead to a decrease in flufenacet sensitivity.

Among the set of 218 contigs with significantly lower expression levels, the largest group of contigs with similar BLASTx annotations contained contigs coding for receptor-like kinases, which may play a role in signal transduction or the regulation of transcription factors. In addition, each three of these contigs were annotated as mitochondrial elongation factors and GSTs, while all other annotations occurred less frequently. Contigs annotated as the typical housekeeping gene actin (e.g., contigs alomy11359 or alomy027178) were not among the set of differentially expressed genes and typically showed homogenous expression patterns.

Based on the results of the interaction study with GST inhibitors (Figure 2) and previous findings on the detoxification pathway of flufenacet in A. myosuroides (Dücker et al. 2019b), the GSTs with significantly higher expression levels in population Kehdingen2 were selected as candidate genes. The open reading frames of seven GSTs with significantly higher levels of expression were identified. Based on their amino acid sequences, four were identified as GSTs belonging to class tau, two as phi-class GSTs, and one as a thetaclass GST (Table 1). As two of the contigs coding for tau-class GSTs had an amino acid similarity of $100 \%$, only the longer contig was chosen for further analysis.

The analysis of the expression patterns of the six candidate GSTs revealed that all of them were more highly expressed in populations Kehdingen1 and Kehdingen2. In all six cases, the level of expression was significantly higher in population Kehdingen2 in comparison to the sensitive population Herbiseed-S. Only the level of expression of GST2 (tau) was also significantly higher in population Kehdingen 1 in comparison to the sensitive populations. This suggests a generally higher expression level of detoxificationrelated genes or pathway(s) in population Kehdingen2 and is in 


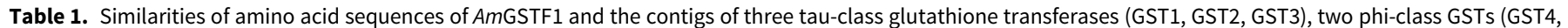
GST5), and a theta-class isoform (GST6) with their contig names as published in the reference transcriptome by Gardin et al. (2015).

\begin{tabular}{|c|c|c|c|c|c|c|c|c|c|}
\hline \multicolumn{3}{|c|}{ Contig description } & \multicolumn{7}{|c|}{ Sequence similarities between contigs of different GSTs \% } \\
\hline Contig name & GST class & GST name & GST1 & GST2 & GST3 & GST4 & GST5 & GST6 & AmGSTF1 \\
\hline Alomy040330 & Tau & GST1 & - & & & & & & \\
\hline Alomy042368 & Tau & GST2 & 74.2 & - & & & & & \\
\hline Alomy056271 & Tau & GST3 & 52.7 & 61.9 & - & & & & \\
\hline Alomy071497 & Phi & GST4 & 22.8 & 25.9 & 28 & - & & & \\
\hline Alomy085325 & Phi & GST5 & 20.6 & 15.7 & 19.9 & 5.7 & - & & \\
\hline ALomy020490 & Theta & GST6 & 22.9 & 24.8 & 14.7 & 10.1 & 5 & - & \\
\hline - & Phi & AmGSTF1 & 31 & 35.5 & 31.3 & 44.5 & 27 & 19.2 & - \\
\hline
\end{tabular}
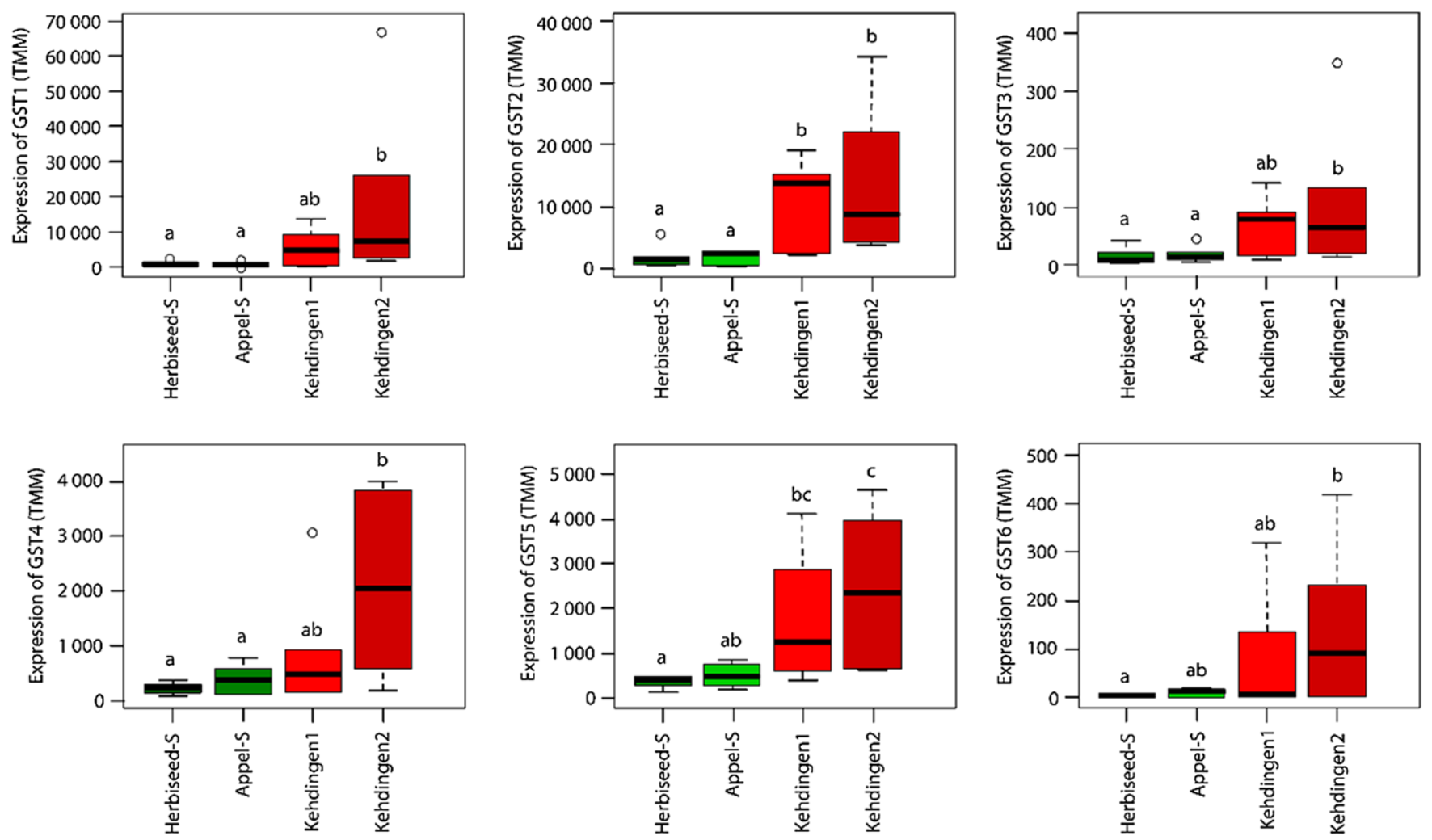

Figure 4. Expression of three tau-class glutathione transferases (GST1, GST2, GST3), two phi-class GSTs (GST4, GST5), and a theta-class isoform (GST6) differentially expressed in the sensitive Alopecurus myosuroides populations Herbiseed-S and Appel-S and the populations Kehdingen1 and Kehdingen2 with reduced flufenacet efficacy. Different letters indicate significant differences between populations (false discovery rate $\leq 0.05$ ). TMM, trimmed mean of $M$ values.

accordance with the resistance level characterized in the doseresponse bioassay. However, the expression levels differed from contig to contig. While GST1 (tau), GST2 (tau), GST4 (phi), and GST5 (phi) achieved relatively high expression levels, particularly in individuals of population Kehdingen2, GST3 (tau) and GST6 (theta) were expressed at low levels, with TMM values below 100 (Figure 4). Finally, AmGSTF1, which is frequently associated with metabolic herbicide resistance in A. myosuroides, was not among the candidate GSTs (sequence similarities $<44.5 \%$ with local alignment; Table 1). This isoform was the only commonly upregulated gene in a set of multiple-resistant $A$. myosuroides analyzed in an RNA-seq experiment conducted by Tétard-Jones et al. (2018), indicating upregulation of different pathways in different multipleresistant populations. Yet all of these genes may play minor or major roles in flufenacet detoxification by glutathione conjugation, as indicated by the inhibitor tests and the metabolites identified in A. myosuroides and flufenacet-tolerant crops (Bieseler et al. 1997; Dücker et al. 2019b).

However, in addition to their classical function of glutathione conjugation and their role in detoxification, GSTs can fulfill several other roles in plants that may indirectly decrease the activity of herbicides in A. myosuroides plants. These roles include their function as ligandins (e.g., for the auxin indole-3-acetic acid; Bilang and Sturm 1995; Sylvestre-Gonon et al. 2019) and additionally include their glutathione-dependent hyperoxidase activity (Axarli et al. 2009). The catalytic and noncatalytic functions of GSTs are involved in tolerance to abiotic stresses, regulation of antioxidants, pathogen defense, or signaling (Cummins et al. 2013; Gullner et al. 2018; Kumar and Trivedi 2018; Marrs 1996; Nianiou-Obeidat et al. 2017). Thus, it has been confirmed 
that various plant GSTs are induced by environmental factors such as pathogens, xenobiotics, metals, drought, and cold, as well as phytohormone production or oxidative stress, which typically accompany the environmental factors (Gullner et al. 2018; Lallement et al. 2014; Marrs 1996; Nianiou-Obeidat et al. 2017; Sylvestre-Gonon et al. 2019).

The regulation of GST expression in plants can be complex, as multiple transcription start points can be present (Thatcher et al. 2007) and multiple regulatory elements can be present in the promoter regions of GSTs. For example, in the promoter region of the rice GST OsGSTL2, several stress-regulated cis elements such as Box-W1, EIRE, or LTR or elements responding to phytohormones such as TCA, CGTCA-motif, or ERE have been found. Similarly, the presence of $\mathrm{ABRE}$ and MYB in the promoter region of a tau-class GST of the succulent Salicornia brachiata Roxb. (Tiwari et al. 2016) indicates concurrent binding of different transcription factors (Hu et al. 2011).

The regulation of GST expression in weeds has so far only been described in a limited number of detailed studies (Brazier-Hicks et al. 2018; Wei et al. 2019). There is, however, evidence that posttranscriptional modifications play a role in resistance-related gene expression (Nandula et al. 2019; Tétard-Jones et al. 2018).

The regulation of GST expression, in particular the roles of the significantly more highly and less expressed transcription factors and their potential to interact with DNA binding domains for the expression of the identified candidate GSTs, remains to be investigated in the A. myosuroides populations studied here, which would require analyses of the genomic sequences. Further analysis of epigenetic modifications (e.g., methylation) of the GST promoters, which could modify gene expression (Gressel 2009; Schnekenburger et al. 2014), is also required.

Differentially expressed genes such as the set of receptor-like kinases with significantly lower expression levels may play a role in differential flufenacet susceptibility. With more than 600 isoforms identified in Arabidopsis and more than 1,100 isoforms identified in rice, these kinases belong to the largest family of receptors in plants (Shiu et al. 2004). The functions of these signaling proteins range from pathogen response via morphological development to functions involved in processes such as selfincompatibility (Morillo and Tax 2006; Shpak et al. 2004; Stein et al. 1991; Tang et al. 2017). Receptor-like kinase-mediated signaling can activate and repress signaling pathways and is affected by multiple interactions of extracellular domains (Jaillais et al. 2011; Smakowska-Luzan et al. 2018). While some kinases can affect gene expression (e.g., by phosphorylation of transcription factors; Sirichandra et al. 2010), the role of these differentially expressed receptor-like kinases remains unclear.

Finally, the reduction of flufenacet detoxification in the presence of the GST inhibitors ethacrynic acid and tridiphane and the detection of six significantly more highly expressed GSTs provide further evidence concerning the role of GSTs in the detoxification of flufenacet and, thereby, the similarity of mechanisms in crops and grass weeds. Additional research is needed to validate the detected candidate genes involved in reduced flufenacet sensitivity in A. myosuroides. The availability of full cDNA sequences and genomic sequences will allow characterization of the biochemical function of the selected genes as well as a better understanding of regulation of their expression (Ravet et al. 2018). This will provide a better understanding of the evolution of herbicide resistance and the prediction of cross-resistance patterns. The implementation of agricultural practices to slow down or even avoid the evolution of flufenacet resistance is becoming increasingly important. This is particularly the case because the maximum field rate of flufenacet may be significantly reduced, as proposed in Europe, increasing the potential risk of sublethal dose rates selecting for non-target site resistance in the future. The knowledge of the detoxification pathways of the different herbicides and the genes involved will help to (1) set specific resistance diagnostics and (2) define the best mixture strategies, either by application of tank mixtures, premixed products, or sequential treatments.

Acknowledgments. The authors would like to thank Alberto Collavo and Joachim Kaiser for providing seed material and Falco Peter, Julia Unger, and Thomas Schubel for support with bioassays and sample shipment. The authors would like to express their great appreciation to Thomas Wolf, Michael Kohnen, Hans-Jürgen Albrecht, and Francesco Pulitano for providing the computational basis for bioinformatic analyses. Finally, the authors thank Andreas von Tiedemann, Lothar Lorentz, Anita Küpper, and Ralf Nauen for scientific discussions and Bayer AG, Crop Science Division for funding and access to equipment. No conflicts of interest have been declared.

\section{References}

Axarli I, Dhavala P, Papageorgiou AC, Labrou NE (2009) Crystal structure of Glycine max glutathione transferase in complex with glutathione: investigation of the mechanism operating by the Tau class glutathione transferases. Biochem J 422:247-256

Bailly GC, Dale RP, Archer SA, Wright DJ, Kaundun SS (2012) Role of residual herbicides for the management of multiple herbicide resistance to ACCase and ALS inhibitors in a black-grass population. Crop Prot 34:96-103

Bieseler B, Fedtke C, Neuefeind T, Etzel W, Prade L, Reinemer R (1997) Maize selectivity of FOE 5043: degradation of active ingredient by glutathione$S$-transferases. Pflanzenschutz-Nachrichten Bayer 50:117-140

Bilang J, Sturm A (1995) Cloning and characterization of a glutathione $S$-transferase that can be photolabeled with 5-azido-indole-3-acetic acid. Plant Physiol 109:253-260

Brabham C, Norsworthy JK, Houston MM, Varanasi VK, Barber T (2019) Confirmation of S-metolachlor resistance in Palmer amaranth (Amaranthus palmeri). Weed Technol 33:720-726

Brazier-Hicks M, Knight KM, Sellars JD, Steel PG, Edwards R (2018) Testing a chemical series inspired by plant stress oxylipin signalling agents for herbicide safening activity. Pest Manag Sci 74:828-836

Busi R, Gaines TA, Walsh MJ, Powles SB (2012) Understanding the potential for resistance evolution to the new herbicide pyroxasulfone: field selection at high doses versus recurrent selection at low doses: resistance evolution by high- and low-dose selection. Weed Res 52:489-499

Camacho C, Coulouris G, Avagyan V, Ma N, Papadopoulos J, Bealer K, Madden TL (2009) BLAST+: architecture and applications. BMC Bioinformatics 10:421

Conesa A, Götz S, García-Gómez JM, Terol J, Talón M, Robles M (2005) Blast2GO: a universal tool for annotation, visualization and analysis in functional genomics research. Bioinformatics 21:3674-3676

Cummins I, Wortley DJ, Sabbadin F, He Z, Coxon CR, Straker HE, Sellars JD, Knight K, Edwards L, Hughes D, Kaundun SS, Hutchings S-J, Steel PG, Edwards R (2013) Key role for a glutathione transferase in multiple-herbicide resistance in grass weeds. Proc Natl Acad Sci USA 110:5812-5817

Dücker R, Zöllner P, Lümmen P, Ries S, Collavo A, Beffa R (2019a) Glutathione transferase plays a major role in flufenacet resistance of ryegrass (Lolium spp.) field populations. Pest Manag Sci 75:3084-3092

Dücker R, Zöllner P, Parcharidou E, Ries S, Lorentz L, Beffa R (2019b) Enhanced metabolism causes reduced flufenacet sensitivity in black-grass (Alopecurus myosuroides Huds.) field populations. Pest Manag Sci 75:2996-3004.

Evans AF Jr, O’Brien SR, Ma R, Hager AG, Riggins CW, Lambert KN, Riechers DE (2017) Biochemical characterization of metabolism-based atrazine resistance in Amaranthus tuberculatus and identification of an expressed GST associated with resistance. Plant Biotechnol J 15:1238-1249 
Ezra G, Dekker JH, Stephenson GR (1985) Tridiphane as a synergist for herbicides in corn (Zea mays) and proso millet (Panicum miliaceum). Weed Sci 33:287-290

Gardin JAC, Gouzy J, Carrère S, Délye C (2015) ALOMYbase, a resource to investigate non-target-site-based resistance to herbicides inhibiting acetolactate-synthase (ALS) in the major grass weed Alopecurus myosuroides (black-grass). BMC Genomics 16:590

Gressel J (2009) Evolving understanding of the evolution of herbicide resistance. Pest Manag Sci 65:1164-1173

Gronwald JW, Plaisance KL (1998) Isolation and characterization of glutathione $S$-transferase isozymes from sorghum. Plant Physiol 117:877-892

Gullner G, Komives T, Király L, Schröder P (2018) Glutathione S-transferase enzymes in plant-pathogen interactions. Front Plant Sci 9:1836

Haslam TM, Kunst L (2013) Extending the story of very-long-chain fatty acid elongation. Plant Sci 210:93-107

Hazarika A, Sarkar S, Hajare S, Kataria M, Malik J (2003) Influence of malathion pretreatment on the toxicity of anilofos in male rats: a biochemical interaction study. Toxicology 185:1-8

Heap I (2019) The International Survey of Herbicide Resistant Weeds. http:// www.weedscience.org. Accessed: December 23, 2019

Hu T, He S, Yang G, Zeng H, Wang G, Chen Z, Huang X (2011) Isolation and characterization of a rice glutathione $S$-transferase gene promoter regulated by herbicides and hormones. Plant Cell Rep 30:539-549

Huang Y-C, Huang H-L, Yeh C-N, Lin K-J, Yu C-S (2015) Investigation of brain tumors using 18F-fluorobutyl ethacrynic amide and its metabolite with positron emission tomography. OncoTargets Ther 8:1877

Hull RI, Moss SR (2012) Is the increasing reliance on residual herbicides for Alopecurus myosuroides (black-grass) control sustainable? Asp Appl Biol:25-32

Jaillais Y, Belkhadir Y, Balsemão-Pires E, Dangl JL, Chory J (2011) Extracellular leucine-rich repeats as a platform for receptor/coreceptor complex formation. Proc Natl Acad Sci USA 108:8503-8507

Jain M, Ghanashyam C, Bhattacharjee A (2010) Comprehensive expression analysis suggests overlapping and specific roles of rice glutathione $S$-transferase genes during development and stress responses. BMC Genomics 11:73

Kapoli P, Axarli IA, Platis D, Fragoulaki M, Paine M, Hemingway J, Vontas J, Labrou NE (2008) Engineering sensitive glutathione transferase for the detection of xenobiotics. Biosens Bioelectron 24:498-503

Kumar S, Trivedi PK (2018) Glutathione S-transferases: role in combating abiotic stresses including arsenic detoxification in plants. Front Plant Sci 9:751

Lallement P-A, Brouwer B, Keech O, Hecker A, Rouhier N (2014) The still mysterious roles of cysteine-containing glutathione transferases in plants. Front Pharmacol 5:192

Lamoureux GL, Rusness DG (1986) Tridiphane [2-(3,5-dichlorophenyl)-2(2,2,2-trichloroethyl)oxirane] an atrazine synergist: enzymatic conversion to a potent glutathione $S$-transferase inhibitor. Pestic Biochem Phys 26:323-342

Li D, Gao Q, Xu L, Pang S, Liu Z, Wang C, Tan W (2017) Characterization of glutathione $S$-transferases in the detoxification of metolachlor in two maize cultivars of differing herbicide tolerance. Pestic Biochem Phys 143:265-271

Li H (2013) Aligning sequence reads, clone sequences and assembly contigs with BWA-MEM. arXiv preprint arXiv:13033997 [q-bio.GN]

Marrs KA (1996) The functions and regulation of glutathione $S$-transferases in plants. Annu Rev Plant Biol 47:127-158

Mennan H, Bozoglu M, Isik D (2003) Economic thresholds of Avena spp., and Alopecurus myosuroides in winter wheat fields. Pak J Bot 35:147-154

Monticolo F, Colantuono C, Chiusano ML (2017) Shaping the evolutionary tree of green plants: evidence from the GST family. Sci Rep-UK 7:14363

Morillo SA, Tax FE (2006) Functional analysis of receptor-like kinases in monocots and dicots. Curr Opin Plant Biol 9:460-469

Moss S, Ulber L, den Hoed I (2019) A herbicide resistance risk matrix. Crop Prot 115:13-19

Nandula VK, Riechers DE, Ferhatoglu Y, Barrett M, Duke SO, Dayan FE, Goldberg-Cavalleri A, Tétard-Jones C, Wortley DJ, Onkokesung N, Brazier-Hicks M, Edwards R, Gaines T, Iwakami S, Jugulam M, Ma R (2019) Herbicide metabolism: crop selectivity, bioactivation, weed resistance, and regulation. Weed Sci 67:149-175
Neve P, Powles S (2005) Recurrent selection with reduced herbicide rates results in the rapid evolution of herbicide resistance in Lolium rigidum. Theor Appl Genet 110:1154-1166

Nianiou-Obeidat I, Madesis P, Kissoudis C, Voulgari G, Chronopoulou E, Tsaftaris A, Labrou NE (2017) Plant glutathione transferase-mediated stress tolerance: functions and biotechnological applications. Plant Cell Rep 36:791-805

Parcharidou E (2019) Characterisation of Flufenacet Resistance in Grass Weeds using Bioassays, Molecular and Analytical Tools. M.S. thesis. Göttingen: Georg-August University Göttingen. $77 \mathrm{p}$

Peterson MA, Collavo A, Ovejero R, Shivrain V, Walsh MJ (2018) The challenge of herbicide resistance around the world: a current summary. Pest Manag Sci 74:2246-2259

Ravet K, Patterson EL, Krähmer H, Hamouzová K, Fan L, Jasieniuk M, LawtonRauh A, Malone JM, McElroy JS, Merotto A Jr, Westra P (2018) The power and potential of genomics in weed biology and management. Pest Manag Sci 74:2216-2225

Ritz C, Baty F, Streibig JC, Gerhard D (2015) Dose-response analysis using R. PLoS ONE 10:e0146021

Robinson MD, Oshlack A (2010) A scaling normalization method for differential expression analysis of RNA-seq data. Genome Biol 11:R25

Rosenhauer M, Petersen J (2015) Bioassay development for the identification of pre-emergence herbicide resistance in Alopecurus myosuroides (Huds.) populations. Gesunde Pflanz 67:141-150

Schnekenburger M, Karius T, Diederich M (2014) Regulation of epigenetic traits of the glutathione S-transferase P1 gene: from detoxification toward cancer prevention and diagnosis. Front Pharmacol 5:170

Shiu S-H, Karlowski WM, Pan R, Tzeng Y-H, Mayer KF, Li W-H (2004) Comparative analysis of the receptor-like kinase family in Arabidopsis and rice. Plant Cell 16:1220-1234

Shpak ED, Berthiaume CT, Hill EJ, Torii KU (2004) Synergistic interaction of three ERECTA-family receptor-like kinases controls Arabidopsis organ growth and flower development by promoting cell proliferation. Development 131:1491-1501

Sirichandra C, Davanture M, Turk BE, Zivy M, Valot B, Leung J, Merlot S (2010) The Arabidopsis ABA-activated kinase OST1 phosphorylates the bZIP transcription factor ABF3 and creates a 14-3-3 binding site involved in its turnover. PLoS ONE 5:e13935

Skipsey M, Andrews CJ, Townson JK, Jepson I, Edwards R (1997) Substrate and thiol specificity of a stress-inducible glutathione transferase from soybean. FEBS Lett 409:370-374

Smakowska-Luzan E, Mott GA, Parys K, Stegmann M, Howton TC, Layeghifard M, Neuhold J, Lehner A, Kong J, Grünwald K, Weinberger N, Satbhai SB, Mayer D, Busch W, Madalinski M, et al. (2018) An extracellular network of Arabidopsis leucine-rich repeat receptor kinases. Nature 553:342

Stein JC, Howlett B, Boyes DC, Nasrallah ME, Nasrallah JB (1991) Molecular cloning of a putative receptor protein kinase gene encoded at the self-incompatibility locus of Brassica oleracea. Proc Natl Acad Sci USA 88:8816-8820 Sylvestre-Gonon E, Law SR, Schwartz M, Robe K, Keech O, Didierjean C, Dubos C, Rouhier N, Hecker A (2019) Functional, structural and biochemical features of plant serinyl-glutathione transferases. Front Plant Sci 10:608

Tang D, Wang G, Zhou J-M (2017) Receptor kinases in plant-pathogen interactions: more than pattern recognition. Plant Cell 29:618-637

Tétard-Jones C, Sabbadin F, Moss S, Hull R, Neve P, Edwards R (2018) Changes in the proteome of the problem weed blackgrass correlating with multipleherbicide resistance. Plant J 94:709-720

Thatcher LF, Carrie C, Andersson CR, Sivasithamparam K, Whelan J, Singh KB (2007) Differential gene expression and subcellular targeting of Arabidopsis glutathione $S$-transferase F8 is achieved through alternative transcription start sites. J Biol Chem 282:28915-28928

Timmerman KP (1989) Molecular characterization of corn glutathione $S$-transferase isozymes involved in herbicide detoxication. Physiol Plantarum 77:465-471

Tiwari V, Patel MK, Chaturvedi AK, Mishra A, Jha B (2016) Functional characterization of the tau class glutathione-S-transferases gene (SbGSTU) promoter of Salicornia brachiata under salinity and osmotic stress. PLoS ONE 11:e0148494 
Trenkamp S, Martin W, Tietjen K (2004) Specific and differential inhibition of very-long-chain fatty acid elongases from Arabidopsis thaliana by different herbicides. Proc Natl Acad Sci USA 101:11903-11908

Wagner U, Edwards R, Dixon DP, Mauch F (2002) Probing the diversity of the Arabidopsis glutathione $S$-transferase gene family. Plant Mol Biol 49:515-532
Wei L, Zhu Y, Liu R, Zhang A, Zhu M, Xu W, Lin A, Lu K, Li J (2019) Genome wide identification and comparative analysis of glutathione transferases (GST) family genes in Brassica napus. Sci Rep 9:1-13

Yu Q, Powles S (2014) Metabolism-based herbicide resistance and crossresistance in crop weeds: a threat to herbicide sustainability and global crop production. Plant Physiol 166:1106-1118 